\title{
Gaining insights on the influence of attention, anxiety, and anticipation on pain perception
}

\section{Ellentika Chayadi \\ Bridget L McConnell}

Psychology Department, James Cook University, Singapore
Correspondence: Bridget L McConnell James Cook University, 149 Sims Drive, Singapore 387380

Tel +65 67093733

Fax +65 67093889

Email bridget.mcconnell@jcu.edu.au
This article was published in the following Dove Medical Press journal: Journal of Pain Research

\begin{abstract}
Purpose: This article highlights the influence of attention and pain anticipation on pain attenuation. Pain-related trait anxiety was found to moderate the effect that attention strategies impose on pain perception. This article may contribute to clinical treatments quality, where pain attenuation effect is desired.
\end{abstract}

Participants and methods: One hundred seven participants, comprising of 72 (67\%) females and $35(33 \%)$ males between the age of 17 and $48(\mathrm{M}=22.6, \mathrm{SD}=4.36)$, were used in the analysis. The current study measured the effect of pain anticipation and attention on three aspects of pain perception: threshold, tolerance, and perceived pain intensity. Pain anticipation was manipulated by varying the amount of information given to participants about a future pain stimulus. Attention was manipulated through a sensory focusing task and a distraction task. Participants were randomized into 1) InfoControl group with distraction task trial (n=30), 2) InfoControl group with attention to pain trial $(n=26), 3)$ InfoExtra group with distraction task trial $(n=26)$, or 4) InfoExtra group with attention to pain trial $(n=25)$. The pain stimulus was delivered in a form of heat. The moderating effects of pain-related trait anxiety on these variables were also investigated using Pain Anxiety Symptom Scale Short Form.

Results: Two structural equation models revealed that anticipation is not a predictor of pain perception and neither did it interact with pain-related trait anxiety. However, attention strategies do significantly relate to pain perception. Furthermore, pain-related anxiety was a significant moderator of attention and pain attenuation. These findings imply that the effectiveness of attention strategies in attenuating pain is affected by individuals' pain-related trait anxiety.

Conclusion: The results suggest the importance of appointing the appropriate attention strategy to different individuals with varying level of trait anxiety. Future explorations are necessary to develop a more specific understanding on the nature of information and distractions on pain perception.

Keywords: distractions, pain attenuation, sensory focusing, trait anxiety

\section{Introduction}

The experience of pain is subjective and modifiable through a broad range of cognitive factors, including attention and pain anticipation. ${ }^{1,2}$ The current understanding of attention and its pain modulating effect revolves around Kahneman' ${ }^{3}$ model of limited attentional resource. ${ }^{4}$ According to this model, when a substantial amount of attention is devoted to a primary task, it leaves limited attentional resources for secondary tasks. ${ }^{3}$ Therefore, the presence of painful stimuli in conjunction with cognitive tasks causes the two stimuli to compete for attention, which results in a suppressed pain processing. ${ }^{5}$ This suggests that engagement in cognitive tasks results in the inhibition of attention 
and neuronal responses to painful stimuli. ${ }^{2}$ Indeed, there are lots of studies reporting reduced pain perception when participants are distracted. ${ }^{6-9}$ For example, a study by Schmitt et al reported lower perceived pain intensity ratings when burn patients were distracted using a virtual reality system during their therapy sessions relative to a control condition. ${ }^{10}$ In addition, neuroimaging studies have reported an increase in the activity of periaqueductal gray matter, best known to contribute to the attenuation of pain, when participants are distracted from a painful stimulus. ${ }^{11-15}$ Together, these studies suggest that pain is perceived to be less intense when individuals are distracted (cf. Van Ryckeghem et al). ${ }^{9,16-19}$ Consequently, distractions are commonly utilized to attenuate patients' pain perception in medical settings. . $^{1,2,20}$

However, these results are complicated by other studies, which show that attending to the noxious stimulus reduced pain perception. ${ }^{2,21}$ For example, Keogh et $\mathrm{al}^{22}$ found that male participants reported lower pain intensity when they were instructed to attend to the painful cold pressor stimulus relative to when they were distracted from it. A similar finding was observed by Nouwen et al, ${ }^{23}$ when participants were asked to continually verbalize their perceived physical sensation during an exposure to cold-induced pain. Although some studies found non-significant interaction between anxiety and the perception of pain, ${ }^{16}$ the inconsistencies of these findings have mostly been attributed to pain-related trait anxiety. ${ }^{24-28}$ The superiority of distraction in pain attenuation is evident mainly in individuals with low pain-related trait anxiety (but see Schreiber). ${ }^{28-30}$ Highly anxious individuals, however, often engage in hypervigilance, which creates an attentional bias as they selectively attend to pain-related stimuli over neutral stimuli. ${ }^{27,31-33}$ Consequently, the attentional bias leads to difficulty in disengaging their attention from threatening stimuli, thus rendering distraction tasks less effective in reducing pain perception. ${ }^{34}$

Sensory focusing strategies are believed to be better suited for individuals with high pain-related anxiety. ${ }^{35}$ According to Leventhal's dual processing theory, information can be processed through subjective/emotional or objective/sensation ways. ${ }^{36}$ Subjective processing focuses on the emotional aspects of pain perception, such as worry, which induces pain catastrophizing behavior that can worsen the experience of pain. ${ }^{21,24}$ Objective processing, however, focuses on the actual pain sensation, and it helps to reduce pain catastrophizing. This model predicts that focusing on the sensory aspects of a painful stimulus allows for an objective evaluation of the sensation and prevents activation of emotional distress. ${ }^{21,24}$ This is consistent with findings from Blitz and Dinnerstein. ${ }^{37}$
Although they did not measure trait anxiety, they did show that instructing participants to dissociate the sensory and emotional aspects of a cold stimulus and focus only on the sensory aspect of a cold stimulus resulted in reduced pain perception. It is interesting that the authors interpreted this technique as a distraction manipulation, since participants were distracted from the emotional aspects of the painful stimulus by focusing on the sensory features. Indeed, it is possible that highly engaging distractor tasks will produce an attenuation in pain perception in highly anxious individuals, but this is dependent upon the level of stimulation and engagement offered by the task. ${ }^{28,38}$

Another cognitive source of influence on pain perception is how much information individuals know about an upcoming painful stimulus; that is, how well individuals can anticipate pain and what they expect to happen. ${ }^{27,29,39}$ It has long been known that predictability, or rather unpredictability, is a major factor in anxiety. ${ }^{40-42}$ Medical patients who receive information about an upcoming procedure show lower anxiety compared to controls. ${ }^{43-45}$ This is relevant as higher levels of anxiety are associated with more sensitivity or perception of pain. ${ }^{46-50}$ Pain anticipation is typically manipulated by giving a signal for an impending aversive event $^{42}$ or through treatment education. ${ }^{51-53}$ Hospitalized patients who received treatment education reported lower perceived anxiety ratings and lower pain intensity relative to control group patients. ${ }^{51,54,55}$ Similar findings were also found when additional information was given prior to surgery, chemotherapy, and colonoscopy procedures. ${ }^{43,51,56}$ One explanation is that predictability of pain enables one to prepare a behavioral response. ${ }^{29,57}$ This elicits the perception of cognitive control, which reduces anxiety, thereby lowering self-reported pain intensity. ${ }^{27}$

However, in contrast to studies showing analgesic effects, other research reports that pain predictability is associated with pain amplification. ${ }^{53}$ For instance, participants who received a verbal warning about an oncoming pain stimulus reported more pain than those who did not receive the verbal warning. ${ }^{58}$ Similarly, anticipation of excessive discomfort was a significant predictor of patients' higher anxiety ratings and overall discomfort ratings. ${ }^{53}$ One explanation for this result is that anticipation of excessive pain induces higher state anxiety and heightened arousal toward the painful stimulus, which correlates with stronger responses to pain. ${ }^{16}$ This is consistent with findings from Tang and Gibson, ${ }^{46}$ who showed that high trait anxiety individuals perceive more pain than low trait anxiety individuals. Moreover, inducing state anxiety resulted in even more pain perception. These findings suggest 
that negative or exaggerated expectations of pain can amplify pain sensations instead of attenuating them. ${ }^{59}$

Just like trait anxiety is hypothesized to moderate the relationship between attention and pain perception, it is likely that anxiety also interacts with pain anticipation on pain perception. While predictability of threatening stimuli is expected to lower patients' anxiety, which in turn reduces arousal, the same effect may not be generated within highly anxious individuals. ${ }^{36}$ Presenting additional pain-related information to highly anxious individuals may not only induce selective attentional bias but it will also fixate these individuals to this information due to the impaired attentional disengagement. Consequently, this results in the amplification of pain. Considering the scarcity of studies that explore this moderating effect, there is a need to investigate the moderating effect of pain-related trait anxiety on attentional strategy and pain anticipation.

Based on prior research showing that predictability of a painful stimulus reduces pain perception, the current study first hypothesizes that receiving additional information about impending pain will reduce their pain perception. In the current study, pain perception is operationalized as the following three measures: perceived pain intensity, pain threshold (ie, the point at which participants indicate they feel pain), and pain tolerance (ie, ability to withstand pain). Therefore, making the impending pain more predictable is expected to reduce pain intensity ratings, increase pain threshold, and increase pain tolerance relative to a group that does not receive additional information about the pain stimulus. Moreover, the current study anticipates that trait anxiety will interact with pain predictability such that individuals with low pain-related trait anxiety are expected to show pain attenuation when given more information, but individuals with high pain-related trait anxiety are expected to show pain amplification. This is based on high pain-related trait anxiety individuals' tendency to engage in selective attention. ${ }^{27,31-33}$ Therefore, it is hypothesized that the direct relationship between pain anticipation and pain perception is moderated by pain-related trait anxiety.

Based on the previous finding by Schmitt et al, ${ }^{10}$ the current study also anticipates that distraction from a painful stimulus will result in more pain attenuation compared to focusing one's attention on the pain stimulus. Hence, it is hypothesized that participants in the distraction group will experience pain attenuation (ie, higher pain threshold and tolerance, and lower perceived pain intensity) relative to their baseline pain ratings; in contrast, pain amplification is predicted for participants in the attention group. Again, however, this relationship is expected to be moderated by pain-related trait anxiety. $22,29,34,35$ This study hypothesized that participants with low pain-related anxiety will benefit from a distraction manipulation, and they will experience pain attenuation relative to their baseline pain ratings, whereas participants with high pain-related anxiety will experience pain amplification. Participants with low pain-related anxiety in the attention group are expected to experience pain amplification relative to their baseline pain ratings, while participants with high pain-related anxiety will experience pain attenuation.

To investigate these hypotheses, the current study partially replicated the experimental procedures of Thompson et al. ${ }^{60}$ Thompson et al explored the relative effects of sensory focusing and distraction on pain threshold and tolerance on 76 individuals using noxious thermal (cold and heat) stimuli. In their study, Thompson et al analyzed the dependency of these outcomes on anxiety sensitivity, a trait tendency to interpret sensory information as threatening. They also examined if this effect differs between genders. In this study, each participant underwent four trials. The first two trials were administered under the same coping condition (focusing or distraction) using both heat and cold noxious stimuli. The last two trials were administered in the alternative coping condition using both heat and cold stimuli. During the trials with focusing coping condition, participants were told, "I would like you to try to concentrate as much as you can on the physical sensations that the pad produces. Please try to concentrate only on these physical sensations and try to do this throughout the duration of the trial." In the distraction condition, participants were instructed, "I would like you to try and distract yourself from the sensations produced by the pad and try to focus on something else. Please try to do this throughout the duration of the trial." Pain was measured according to pain threshold and pain tolerance.

The current study only partially replicated Thompson et al's experimental procedure as it only used a heat stimulus, and the distraction/attention variable in the current study was between-subjects instead of within, as was done in Thompson et al's experiment. Moreover, the current study improved upon the attentional manipulation to ensure task compliance. In addition to instructing participants to focus or ignore the pain stimulus, the present study ensured participants' actual attentional focus by engaging in one of two tasks - verbalizing the heat sensation or playing a non-anxiety-inducing game. Furthermore, the present study included another index of pain perception. Thompson et al measured pain using pain threshold and tolerance. While these two indices indicate the physical aspects of pain perception, they do not directly 
measure the cognitive perception of pain. Hence, in addition to assessing pain threshold and tolerance, the current study also measured perceived pain intensity. Finally, the current study added an information manipulation and a pain-related trait anxiety questionnaire to assess how these variables influence pain perception.

\section{Participants and methods Setting}

The study was conducted in a private and windowless room $(3.5 \times 3.0 \mathrm{~m})$ within a research laboratory upon receiving approval from the James Cook University's (JCU) Human Research Ethics Committee (HREC). Participants were seated in front of a table that held the apparatuses, including a standard desktop computer with a 23-inch monitor, QWERTY keyboard, and mouse, and the heating pad. The principal investigator (PI) sat beside the participants with her back facing the participants. This was done to ensure minimal distress of being observed in participants. The PI was always present throughout the study to monitor the temperature of the heat stimulus and ensure a strict conformity to the maximum temperature allowed by the HREC.

\section{Participants}

A total of 117 participants from James Cook University, Singapore were recruited for the purpose of this study. Participants were recruited through JCU Singapore research website, JCU SONA system, and snowball sampling by having those who have completed the study to circulate the study's information sheet to those who are interested. Students who required credit points for the completion of their modules were granted credit points for their participation, while the remaining participants were given no incentives. All individuals are eligible to participate in the study; however, individuals with open wounds on their left palm were excluded.

\section{Materials}

\section{Information sheet and informed consent form}

These documents are required by the JCU HREC for all participants. The information sheet informed participants that they would answer a questionnaire about pain anxiety and a demographic questionnaire. They were also informed that they would complete a pain calibration procedure (PCP) with a heat stimulus to measure pain threshold, tolerance, and perceived pain intensity. Additionally, the information sheet indicated that the heat will be increased only to the point of feeling uncomfortable and no more, and that they can expect to experience minor distress and heat sensation, which will not affect their well-being in the long run. These details were reiterated in the informed consent form in which participants acknowledged that they will knowingly participate in a PCP to investigate the effect of pain anticipation, attention, and anxiety on pain perception.

\section{Herpstat I Basic and heating mat}

Thermal stimulation was delivered via a $15 \times 28 \mathrm{~cm}$ rectangular heating mat connected to a proportional thermostat, Herpstat 1 Basic model. Herpstat 1 Basic allows for a precise adjustment of heat necessary to maintain a controllable target temperature ranging from $4^{\circ} \mathrm{C}$ to $65^{\circ} \mathrm{C}$. A detailed technical description of this product can be found in the user manual online http://www.spyderrobotics.com/manuals/herpstat1Basic manual.pdf.

\section{Slither.io}

The distraction task was a slow-paced explorative game conducted on the computer. Players control an avatar resembling a snake or worm, and the objective of the game is to grow the longest snake by consuming pellets or consuming other snakes. The concept in this game is reminiscent of the classic arcade game Snake. This game was chosen due to its slowpaced and low-skill nature, which allows participants with varying gaming abilities to be engaged in the game without inducing any anxiety.

\section{Stopwatch}

A CASIO stopwatch was used to measure the amount of time that participants are able to withstand the pain stimulus. This time was recorded to the nearest second.

\section{Pain Anxiety Symptoms Scale-Short Form (PASS-20)}

The PASS-20 is a self-report rating scale that measures pain-related trait anxiety. ${ }^{61}$ The questionnaire consists of six sub-scales measuring cognitive, fear, escape/avoidance, and physiological aspects of pain-related anxiety. PASS-20 consists of 20 items comprising statements such as "I can't think straight when in pain". Participants were required to indicate how often they identify with the statement on a 6-point Likert scale ( $0=$ "never", $5=$ "always"). The sum of each item in the PASS-20 ranges from 0 to 100 , in which a higher score indicates higher pain-related trait anxiety. The PASS-20 has demonstrated factorial invariance in clinical pain populations as well as in non-clinical populations. Hence, it serves as an appropriate measure of pain-related trait anxiety in the current population of non-clinical sample. 
The PASS-20 has exhibited an excellent internal consistency in previous studies $(\alpha=0.91)$ and correlates highly with its original form $(r=0.95){ }^{62}$ The questionnaire has generally exhibited a high correlation with other widely used self-report pain-related anxiety measures such as the Fear of Pain Questionnaire-III, suggesting a strong convergent validity $(r=0.53) .{ }^{63}$ The excellent internal consistency is also replicated in this sample with a Cronbach's $\alpha$ of 0.89 .

\section{Numeric Rating Scale (NRS)}

An NRS was used to measure the subjective experience of pain intensity and unpleasantness. It includes a 10-point scale ranging from 1 "Not unpleasant at all" to 10 "Most unpleasant pain imaginable". The NRS was used to measure subjective perceived pain intensity of the heat stimulus used in the experiment.

\section{Design}

This study utilized a mixed-subject experimental design with two independent variables (IVs), one quasi-IV, and three dependent variables (DVs). The IVs were participant's anticipation of pain (Info Control vs Info Extra). This IV was manipulated through the amount of information provided to the participants in addition to the information stated in the information sheet. The second IV was attention (Attention vs Distraction), which varied based on whether participants were required to verbally describe the pain they were feeling or play Slither.io. The quasi-IV in this study was pain-related trait anxiety, which was measured as a continuous variable using the PASS-20.

The DVs of this study were as follows: 1) pain threshold, 2) pain tolerance, and 3) perceived pain intensity. Pain threshold was operationalized as the temperature at which participants first feel uncomfortable. The duration of time that participants were willing to withstand their subjective pain threshold was noted as pain tolerance. Perceived pain intensity was verbally measured through the NRS. All of these variables were measured as the change from Phase 3 to Phase 4. Together, these comprised an overall measure of pain perception.

\section{Procedure}

\section{Phase I - Pre-manipulation}

Participants were provided with an information sheet regarding the nature of the study and their rights. Upon reading the information provided and granting their informed consent, participants were asked to complete a printed non-identifiable demographics form and the PASS-20. Afterward, participants were randomly assigned into one of four groups: 1) InfoControl group with distraction task trial $(\mathrm{n}=30), 2)$ InfoControl group with attention to pain trial ( $\mathrm{n}=26), 3)$ InfoExtra group with distraction task trial ( $\mathrm{n}=26)$, or 4$)$ InfoExtra group with attention to pain trial $(n=25)$.

\section{Phase 2 - Info-manipulation}

The amount of information about what to expect in the experiment was manipulated to influence participants' pain anticipation. Participants in Condition InfoControl were not told anything about the experiment except for what was included in the information sheet and informed consent form. Participants in Condition InfoExtra were provided with additional information about the expected discomfort that they may feel during the experiment, specific flow of the procedure, features of the equipment, and the safety of the procedure. All participants in Condition InfoExtra received the following verbal information:

Before we begin the experiment, please be ensured that no the procedure used in this study is safe and has been adapted from other established studies. Your pain threshold and tolerance will be measured using this thermostat called "Herpstat 1 ". Its temperature range is only from $0^{\circ} \mathrm{C}$ to $50^{\circ} \mathrm{C}$, therefore although it can get a little hot, it is still within the safe temperature range for you. This machine will be set at $36^{\circ} \mathrm{C}$ and the temperature will increase slowly. You will feel a slight warm sensation on your hand, which will slowly get warmer. I will strictly follow the ethical guideline and will stop the trial once you have gone beyond the recommended safety temperature or duration. Please notify me instantly when the temperature is no longer comfortable. Please do not wait until it is painful to tell me. After this trial, two minutes break will be given before your pain tolerance is measured. During tolerance trial, I will need you to place your hand on Herpstat 1, which will be set at the temperature that you said you are uncomfortable with. The duration that you are able to withstand this temperature will be measured. Again, please do not try to withstand the heat and withdraw your hand immediately once you feel uncomfortable or pain. It is expected that you will feel uncomfortable and anxious in this experiment but let me just reiterate that this procedure is safe, and no major physical or mental harm will be done to you.

All participants were reminded to notify the PI as soon as they first feel uncomfortable with the pain stimulus. They were also told that they should not experience actual pain; they should stop the experimenter when they feel discomfort. 


\section{Phase 3 - PCP}

PCP was conducted to determine participants' baseline pain threshold, tolerance, and perceived pain intensity rating. Participants were told to place their left palm on the heat mat, which was connected to a thermostat. All trials began at a temperature of $36^{\circ} \mathrm{C}$ and temperature was slowly increased until participants reported feeling uncomfortable. At this point, participants were instructed to immediately withdraw their hand. This temperature was recorded as their baseline pain threshold $\left(\mathrm{T}_{\mathrm{B}}\right)$. Participants were then asked to rate their perceived pain intensity on the NRS. Subsequently, a 2-minute rest period was carried out to prevent habituation of heat stimuli, during which participants were instructed to play slither.io on the computer placed in front of them.

Once the 2-minute rest period was over, the participants were instructed to place their left palm back down on the heat mat. The temperature was set to their baseline pain threshold $\left(\mathrm{T}_{\mathrm{B}}\right)$, and they were again reminded to notify the PI once they first feel uncomfortable. The PI immediately started the stopwatch when participants' left palms covered the mat and stopped the stopwatch once participants reported feeling uncomfortable. The duration of time was recorded as participants' initial pain tolerance. Participants were again asked to rate their perceived pain intensity on the NRS. After this, another 2-minute rest period was carried out, during which participants continued playing Slither.io.

This procedure was repeated twice to obtain the average initial reading of pain threshold $\left(\mathrm{T}_{\mathrm{i}}\right)$, pain tolerance $\left(\mathrm{Tol}_{\mathrm{i}}\right)$, and perceived pain intensity $\left(\mathrm{Int}_{\mathrm{i}}\right)$ rating. According to the UK burn center, a temperature of $80^{\circ} \mathrm{C}$ can cause burn in a very short time. Hence, to ensure participants' safety, trials were discontinued, should participants' threshold exceeded $60^{\circ} \mathrm{C}$, and tolerance exceeded 7 minutes. Please refer to Figure 1 for a visual representation of the PCP.

\section{Phase 4 -Attention manipulation}

This phase followed the same PCP procedure as described in Phase 3 with an additional attention task. Participants in Condition Attention were asked to pay attention to the heat stimulus. To ensure that participants engaged their attention with the heat stimulus, they were asked to verbally describe the sensation that they are feeling. For example, participants received prompts such as, "Describe where you feel the heat is coming from and where it is spreading to". In Condition Distraction, participants were instructed to ignore the heat stimulus and direct their attention to the game, Slither.io. Familiarization of this game was incorporated in the 2-minute rest period that all participants went through in Phase 3. After

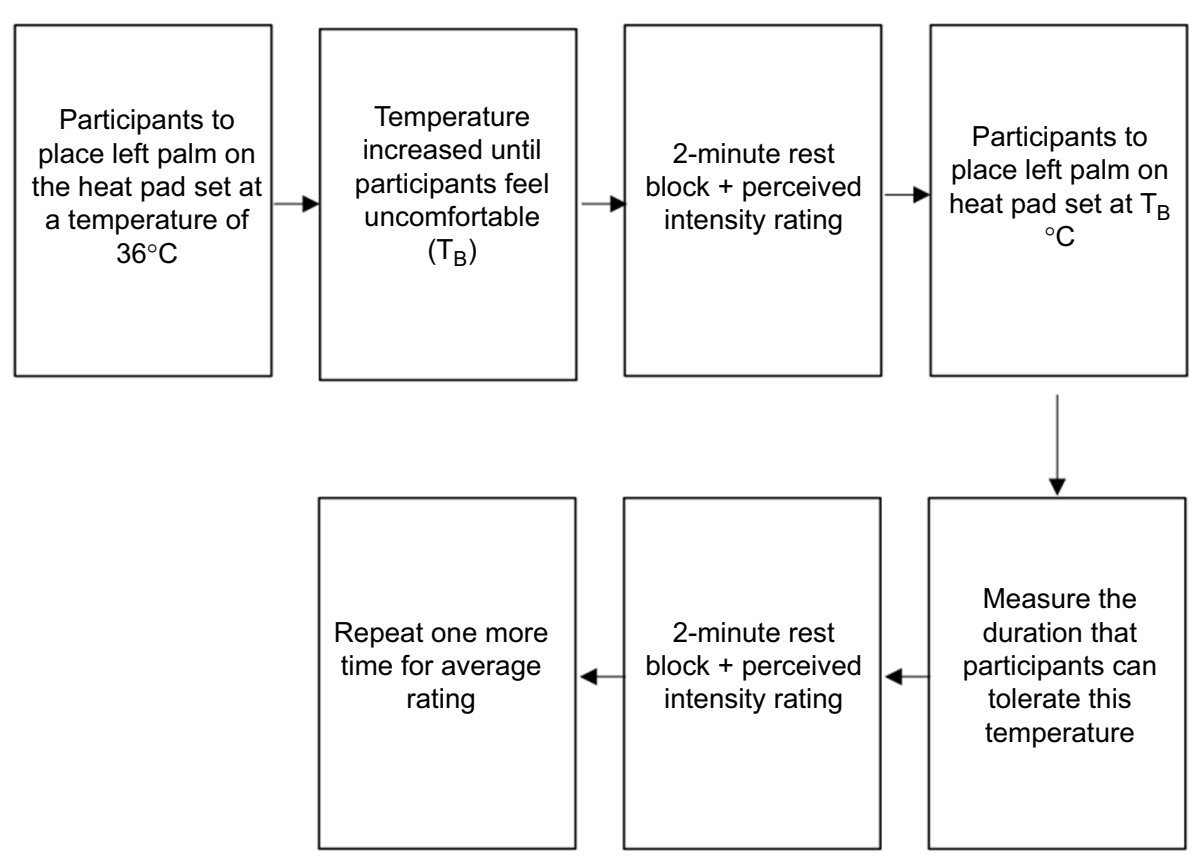

Figure I Summary of PCP.

Notes: This figure depicts the flow of PCP in Phase 3, after participants have undergone information manipulation. The single-headed arrow ( $\rightarrow$ ) denotes the sequence of the procedure from one step to another. $T_{B}$ indicates baseline temperature, at which participants reported feeling uncomfortable.

Abbreviation: PCP, pain calibration procedure. 
ensuring that all participants understood the instructions, the same procedure as described in Phase 3 was conducted while participants engaged in the allocated attention task. Just like in Phase 3, the PCP was conducted twice to obtain an average final score of participants' pain tolerance ( Tol $_{\mathrm{f}}$ ), pain threshold $\left(\mathrm{T}_{\mathrm{f}}\right)$, and perceived pain intensity $\left(\mathrm{Int}_{\mathrm{f}}\right)$. These scores were used to measure the change in pain ratings from Phase 3 (ie, final - initial).

\section{Phase 5 - Debrief}

After the experiment was finished, participants were debriefed about the aims of the study. They were asked if they had felt any lingering discomfort, and none reported any discomfort. Participants were also provided with information for the on-campus clinic if they feel so inclined. Finally, participants were asked not to talk about the experiment to the other students so as to not undermine the information manipulation variable.

\section{Statistical analyses}

Out of the 117 participants, 10 participants were able to withstand thermal stimuli exceeding the ethical guideline of $60^{\circ} \mathrm{C}$ or 7 minutes duration. Consequently, trials for these participants were terminated and their data were excluded. Hence, only 107 remaining participants, comprising of 72 (67\%) females and 35 (33\%) males between the age of 17 and $48(M=22.6, \mathrm{SD}=4.36)$, were used in the analysis. Statistical analyses were conducted using IBM SPSS Statistics for MAC (Version 21.0) ${ }^{64}$ and Amos (Version 21.0), ${ }^{65}$ with $\alpha=0.05$.

Structural equation modeling (SEM) was used to examine the hypotheses of this study. The current investigation measured pain perception through pain threshold, pain tolerance, and perceived pain intensity rating. Model 1 (anticipation model) included predictor variables anticipation and pain-related trait anxiety. Model 2 (attention model) included predictor variables attention and pain-related trait anxiety. Unlike other multivariate analyses, such as multiple linear regression and multivariate analysis of variance (MANOVA), SEM allows for simultaneous analysis of all the variables in the model instead of separately. ${ }^{66}$

The current study proposed the following two models: pain anticipation model (Figure 2) and attention model (Figure 3). The pain anticipation model evaluates the direct effect of additional information on baseline pain perception. It also evaluates the moderating effect of pain-related trait anxiety on the influence of attention and anticipation on pain perception. The attention model evaluates the effect of attention tasks on the change in pain perception. It also evaluates the moderating effect of pain-related trait anxiety on this relationship.

\section{Results}

The assumption of normality was satisfied. The sample also exhibited absence of multicollinearity with variance inflation factor (VIF) and tolerance values $<5$. The data exhibited a sufficiently linear pattern except for the relationship between 1) pain-related trait anxiety and increase in tolerance, and 2) pain-related trait anxiety and increase in perceived pain intensity rating. Because the assumption of linearity was not adequately met, maximum likelihood parameter estimation with bias-corrected bootstrap confidence intervals was used to obtain a more accurate result. The estimation created 500 bootstrap samples and set $95 \%$ bias-corrected CIs. Outliers

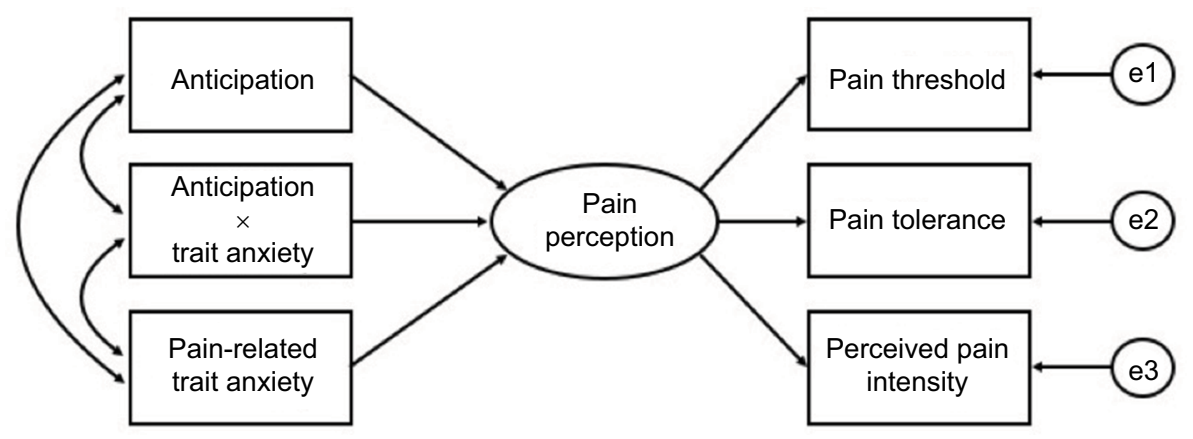

Figure 2 Predicted anticipation model.

Notes: This figure depicts the structural equation modeling used to analyze the influence of pain anticipation, pain-related anxiety, and the moderating effect of pain-related anxiety on pain perception. The rectangles () represent observed variables, while the ellipses () represent the unobserved latent factors. The single-headed arrows with solid lines $(\rightarrow)$ represent the path coefficient for regression of one variable on another. The double-headed arrows $(\leftrightarrow)$ represent covariances or correlations between pairs of variables. Trait anxiety indicates pain-related trait anxiety. " $\times$ ” in Anticipation $\times$ Trait anxiety box indicates the interaction between anticipation and pain-related trait anxiety. el indicates measurement error for pain threshold reading; e2 indicates measurement error for pain tolerance reading; e3 indicates measurement error for perceived pain intensity rating. 


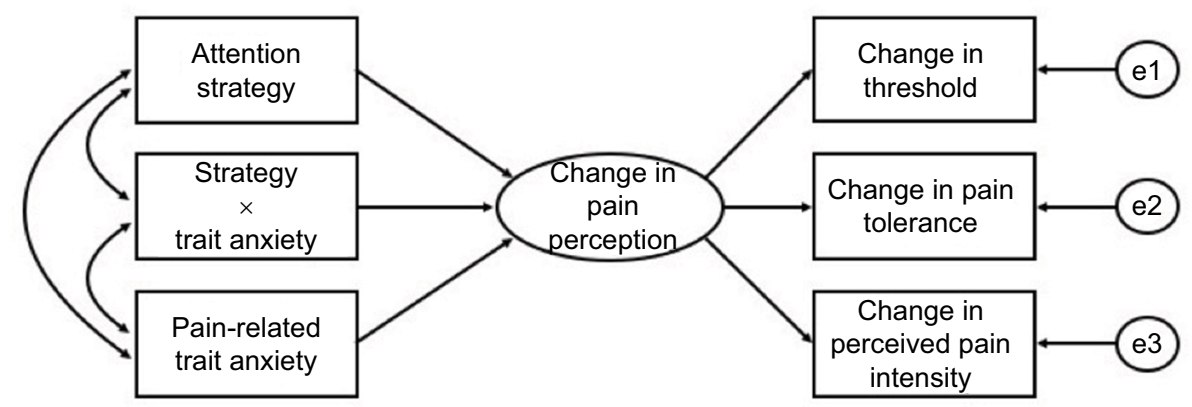

Figure 3 Predicted attention model.

Notes: This figure depicts the structural equation modeling used to analyze the influence of attention strategies, pain-related anxiety, and the moderating effect of painrelated anxiety on the change in pain perception. The rectangles $(\square)$ represent observed variables, while the ellipses $(\boldsymbol{O})$ represent the unobserved latent factors. The single-headed arrows with solid lines $(\rightarrow)$ represent the path coefficient for regression of one variable on another. The double-headed arrows ( $\leftrightarrow$ ) represent covariances or correlations between pairs of variables. Trait anxiety indicates pain-related trait anxiety. " $\times$ " in Strategy $\times$ Trait anxiety box indicates the interaction between attention strategy and pain-related trait anxiety. el indicates measurement error for the change in pain threshold reading; e2 indicates measurement error for the change in pain tolerance reading; $\mathrm{e} 3$ indicates measurement error for the change in perceived pain intensity rating.

in the sample were identified using the squared Mahalanobis distance $\left(D^{2}\right)$. In both models, two participants had $D^{2}$ values of 27.58 and 18.81 , respectively, and a third participant had a $D^{2}$ value of 15.96 . This suggests that there was an outlier in the sample. Hence, a listwise removal, by first removing the most extreme outlier, was performed. This resulted in a closer $D^{2}$ values of the other two participants (18.66 and 17.89). Since there was no longer extreme difference, listwise removal of cases was stopped with only one case removed from InfoExtra group with attention to pain trial. This left the sample at 106 participants $(M=22.6, \mathrm{SD}=4.36)$.

Although this is not a required assumption to be met, it is important to note that $67 \%$ of the sample was females. To ensure that there is no gender bias in the sample, MANOVA was conducted to identify if there is a significant difference in the pain ratings between the two groups. Results revealed that there was no significant difference between the two groups in terms of the averaged scores obtained in Phase 3 and change in pain threshold, pain tolerance, and perceived pain intensity rating $(P>0.05)$. This suggests that there is no gender bias in the sample.

It is important to note that low pain perception is defined here as a lower ability to perceive pain stimulus. This is indicated by higher pain threshold, higher pain tolerance, and lower perceived pain intensity scores. In contrast, high pain perception is defined as a higher ability to perceive pain. This is indicated by lower pain threshold, lower pain tolerance, and higher perceived pain intensity scores.

\section{Anticipation model}

The bootstrapped regression weight estimates in the SEM indicated that information is not a predictor of pain perception
$(B=-0.28, \mathrm{SE}=0.44,95 \% \mathrm{CI}:-1.01,0.62, P>0.05)$, but painrelated trait anxiety is a significant predictor of pain perception $(B=-0.72, \mathrm{SE}=0.33,95 \% \mathrm{CI}:-1.23,-0.75, P<0.05)$. Pain threshold, pain tolerance, and perceived pain intensity were also significant indicators of pain perception, all $P_{\mathrm{s}}<0.05$. The negative regression weight indicates that individuals with lower PASS-20 scores are more likely to have higher pain threshold, higher pain tolerance, and a lower perceived pain intensity, which translates to low ability to perceive pain stimulus (low pain perception).

The interaction effect between information and painrelated trait anxiety was non-significant $(B=0.81, \mathrm{SE}=0.48$, $95 \% \mathrm{CI}:-0.13,1.65, P>0.05)$. A descriptive summary of this model can be found in Table 1 .

Several fit indices were used to evaluate the models: the $\chi^{2}$ goodness-of-fit statistic, relative chi-square $\left(\chi^{2} / d f\right)$, the

Table I Summary of anticipation model

\begin{tabular}{|c|c|c|c|c|}
\hline \multirow[t]{2}{*}{ Path } & \multirow[t]{2}{*}{ B } & \multirow[t]{2}{*}{ SE } & \multicolumn{2}{|l|}{$95 \% \mathrm{Cl}$} \\
\hline & & & Lower & Upper \\
\hline $\begin{array}{l}\text { Pain perception } \leftarrow \text { Anticipation } \\
\text { group }\end{array}$ & -0.28 & 0.44 & -1.01 & 0.62 \\
\hline $\begin{array}{l}\text { Pain perception } \leftarrow \text { Pain-related } \\
\text { anxiety }\end{array}$ & $-0.72 *$ & 0.33 & -1.23 & -0.75 \\
\hline $\begin{array}{l}\text { Pain perception } \leftarrow \text { Anticipation } \\
\times \text { anxiety }\end{array}$ & 0.81 & 0.48 & -0.13 & 1.65 \\
\hline $\begin{array}{l}\text { Pain threshold } \leftarrow \text { Pain } \\
\text { perception }\end{array}$ & 1.00 & & 1.00 & 1.00 \\
\hline $\begin{array}{l}\text { Pain tolerance } \leftarrow \text { Pain } \\
\text { perception }\end{array}$ & $20.1^{*}$ & 34.02 & 1.56 & 63.6 \\
\hline Pain intensity $\leftarrow$ Pain perception & $0.04 *$ & 0.40 & -0.29 & 0.71 \\
\hline
\end{tabular}

Notes: $\mathrm{n}=106 . * \mathrm{P}<0.05$.

Abbreviations: $B$, standardized beta coefficient; SE, standard error; $95 \% \mathrm{Cl}$, lower and upper bounds of $95 \%$ confidence interval. 
goodness-of-fit index (GFI), the comparative fit index (CFI), Tucker-Lewis index (TLI), root mean square residual (RMR), standardized root mean square residual (SRMR), and the root mean square error of approximation (RMSEA). A model is considered to have very good fit if the $\chi^{2}>0.05$, the GFI, TLI, and CFI $>0.95$, and the RMSEA $<0.05$, and SRMR $<0.08 .{ }^{67}$

The general model fit indices of the effects of pain anticipation, and pain-related trait anxiety on pain perception revealed $\chi^{2}=17.70, d f=6, P<0.05, \chi^{2} / d f=2.95$, GFI $=0.95$, $\mathrm{RMR}=1.04, \mathrm{SRMR}=0.13, \mathrm{TLI}=0.74, \mathrm{CFI}=0.89$, RMSEA $=0.13$. This suggests that, although close to the acceptable range, the overall fit of the anticipation model was poor and cannot be used to predict the effect of pain anticipation on pain perception.

\section{Attention model}

The change in pain perception was measured by subtracting the Phase 3 pain ratings from the conditioned pain ratings obtained in Phase 4 (Ratings change = Final rating - Initial rating). Hence, a positive change indicates pain attenuation, while negative change indicates pain amplification. The regression weight estimate indicated that attention tasks can significantly predict change in pain perception $(B=2.78, \mathrm{SE}$ $=0.38,95 \% \mathrm{CI}: 2.04,3.3, P<0.05)$.

Further analyses using MANOVA were conducted to examine the direction of change in pain perception. The analysis revealed a significant effect of attention tasks on the change in pain perception, $F(1,102)=24.82, P<0.05$, $\eta^{2} P=0.42$. It also showed that individuals who were engaged in distraction tasks $(\mathrm{n}=55)$ had a positive change in threshold $(M=1.95, \mathrm{SD}=1.83)$ and tolerance $(M=50.20, \mathrm{SD}=63.10)$, and a negative change in perceived pain intensity $(M=-0.91$, $\mathrm{SD}=1.08$ ). Individuals who were told to pay attention to the pain stimuli $(\mathrm{n}=51)$ scored a negative change in threshold $(M=-0.87, \mathrm{SD}=2.23)$ and tolerance $(M=-27.67, \mathrm{SD}=59.99)$, and a positive change in perceived pain intensity $(M=0.65, \mathrm{SD}$ $=1.33$ ). This suggests that distraction during the procedure attenuated pain, while attention to the task amplified the perception of pain. Refer to Table 2 for a descriptive summary.

The interaction effect between attention task and painrelated trait anxiety was significant $(B=-0.28, \mathrm{SE}=0.07,95 \%$ $\mathrm{CI}:-1.36,-1.88, P<0.05)$. This interaction effect is depicted in Figure 4. These results suggest that individuals with high pain-related anxiety had a positive change in pain perception when they were asked to pay attention to the pain stimuli (ie, pain attenuation), whereas individuals with low pain-related anxiety had a negative change (ie, pain amplification) in pain perception. However, when distracted from the stimuli, all
Table 2 Direction of change in pain perception in attention groups

\begin{tabular}{|l|l|l|l|l|}
\hline Pain perception measures & \multicolumn{2}{l|}{$\begin{array}{l}\text { Sensory } \\
\text { focusing }\end{array}$} & \multicolumn{2}{l|}{ Distraction } \\
\cline { 2 - 5 } & M & SD & M & SD \\
\hline Change in pain threshold & -0.87 & 2.23 & 1.95 & 1.83 \\
Change in pain tolerance & -27.67 & 59.99 & 50.2 & 63.10 \\
Change in pain intensity rating & 0.65 & 1.33 & -0.91 & 1.08 \\
\hline
\end{tabular}

Notes: $n=106$. Sensory focusing refers to Condition Attention in which participants verbally described the sensations of pain they were experiencing. Distraction refers to Condition Distraction in which participants played a game while undergoing the pain calibration procedure. All values indicate the change from baseline.

Abbreviation: $M$, group mean.

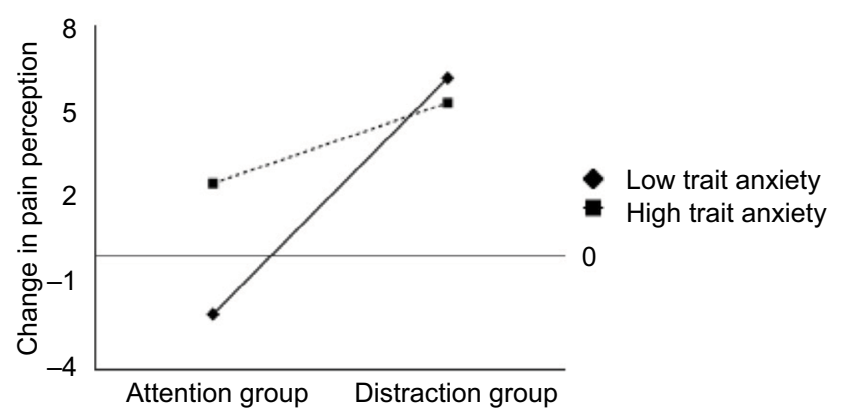

Figure 4 Interaction between attention task and pain-related trait anxiety. Notes: The change in pain perception was measured as final - initial rating of the respective pain perception ratings. Participants in attention group $(n=5 I)$ were told to pay attention to and verbalize the heat sensation that they felt on their palm. Participants in the distraction group $(n=55)$ were told to engage in a game presented on a computer in front of them.

individuals experienced a positive change in pain perception. There was a significant trend for individuals with low painrelated anxiety to have a greater change in pain perception relative to those with higher pain-related anxiety $(B=-1.06$, $\mathrm{SE}=0.29,95 \% \mathrm{CI}:-1.59,-0.41, P<0.05)$. The descriptive summary of the attention model can be found in Table 3 .

The general model fit indices of the effects of attention tasks, and pain-related trait anxiety, on change in pain perception revealed $\chi^{2}=7.56, d f=6, P>0.05, \chi^{2} / d f=1.26$, GFI $=0.98, \mathrm{RMR}=1.03, \mathrm{SRMR}=0.13, \mathrm{TLI}=0.99, \mathrm{CFI}=0.99$, RMSEA $=0.05$. This suggests that the fit of the attention model was good.

\section{Discussion}

In this experiment, we manipulated anticipation and attention to see how each of these variables interacts with pain-related trait anxiety on pain perception. Anticipation and attention were tested separately in SEMs. The results did not support a role for anticipation in predicting pain perception, and nor did this variable interact with trait anxiety. That is, there 
Table 3 Summary of attention model

\begin{tabular}{|c|c|c|c|c|}
\hline \multirow[t]{2}{*}{ Path } & \multirow[t]{2}{*}{ B } & \multirow[t]{2}{*}{ SE } & \multicolumn{2}{|l|}{$95 \% \mathrm{Cl}$} \\
\hline & & & Lower & Upper \\
\hline $\begin{array}{l}\text { Pain perception } \leftarrow \text { Attention } \\
\text { group }\end{array}$ & $2.78^{*}$ & 0.38 & 2.04 & 3.34 \\
\hline $\begin{array}{l}\text { Pain perception } \leftarrow \text { Pain-related } \\
\text { anxiety }\end{array}$ & $-1.06 *$ & 0.29 & -1.59 & -0.41 \\
\hline $\begin{array}{l}\text { Pain perception } \leftarrow \text { Attention } \times \\
\text { anxiety }\end{array}$ & $-0.28 *$ & 0.07 & -1.36 & -1.88 \\
\hline Pain threshold $\leftarrow$ Pain perception & 1.00 & & 1.00 & 1.00 \\
\hline Pain tolerance $\leftarrow$ Pain perception & $29.82 *$ & 3.16 & 25.76 & 35.09 \\
\hline Pain intensity $\leftarrow$ Pain perception & $-0.52 *$ & 0.07 & -0.75 & -0.37 \\
\hline
\end{tabular}

Notes: $\mathrm{n}=106 . * \mathrm{P}<0.05$.

Abbreviations: $B$, standardized beta coefficient; SE, standard error; $95 \% \mathrm{Cl}$, lower and upper bounds of $95 \% \mathrm{Cls}$.

was not a significant direct effect between having more information about the pain stimulus and subsequent pain perception. Hence, our hypotheses were rejected. It is perhaps worth mentioning that, albeit non-significant, the negative regression weight between anticipation and pain perception suggests that additional information correlated with higher pain perception. That is, we observed a trend toward a nocebo effect rather than a placebo effect. ${ }^{46,52,53,58}$ This is contrary to research showing that having more information reduced pain perception.

The moderating effect of pain-related trait anxiety was also investigated. The direct relationship between anticipation and anxiety and the interaction between anticipation and anxiety on pain perception were non-significant. Thus, the proposed hypothesis that the direct relationship between pain anticipation and pain perception is moderated by painrelated trait anxiety was also rejected. However, there was a significant relationship between pain-related trait anxiety and pain perception, which is supportive of other studies that show a positive relationship between anxiety and pain perception. ${ }^{47,68}$

We note that the results of the anticipation model should be considered with caution. The strength of our manipulation was limited due to the constraints placed on us by the ethics committee. All participants were required to be briefed about the pain stimulus and what to expect. Consequently, participants in both groups received similar information with regards to the heat stimulus. This is discussed further in section "Limitations and future directions".

The results in the attention model revealed that the distraction task generally attenuated pain while sensory focusing amplified the experience of pain. In line with Kahneman's ${ }^{3}$ limited attention model, the presence of distractor in conjunction with painful stimulus resulted in the two stimuli to competing for attention. With the instruction to ignore the heat stimulus and focus on the distractor, the distractor became the primary task. Consequently, participants' attention toward the painful stimulus, a secondary task, was diminished. This then inhibited the perception of pain sensation, which resulted in pain attenuation. ${ }^{5}$ On the contrary, when participants were instructed to focus on the painful sensation, complete attention was presumably devoted to the painful sensation. As a result, participants were more cognizant of the painful stimulus; they reported lower pain thresholds and tolerance and higher perceived pain intensity ratings. This result is consistent with several previous studies, which found that, relative to a control condition, individuals who were distracted from the painful stimulus showed less pain perception. ${ }^{7,9,15,69,70}$ Our results also showed that, compared to individuals who were distracted from the pain stimulus, individuals who attended to the pain stimulus reported stronger pain experience. This is consistent with the results of Roelofs et $\mathrm{al}^{36}$ who also showed distraction reduced pain ratings and sensory focusing increased pain ratings in low-fear individuals.

In line with other research, we predicted that the effectiveness of the attention strategies would differ based on pain-related trait anxiety. ${ }^{36}$ Indeed, our results showed that pain-related trait anxiety was a significant moderator, such that sensory focusing was related to pain attenuation for participants with high pain-related trait anxiety, but it predicted pain amplification for participants with low painrelated anxiety. This result is consistent with the studies by Roelofs et $\mathrm{al}^{36}$ and Blitz and Dinnerstein, ${ }^{37}$ and it is in line with Leventhal's ${ }^{71}$ dual processing theory. Instructing highly anxious individuals to focus on the heat sensation allowed for an objective evaluation of the heat sensation and separated the physical sensation from the emotional sensation. ${ }^{21,36}$ Consequently, the objective sensory focus helped to attenuate the painful experience in highly anxious individuals. In some sense, this could in fact be thought of as a distractor task since the participant was distracted from the emotional aspects of the stimulus by focusing on the physical aspects. This is the explanation offered by Blitz and Dinnerstein, ${ }^{37}$ and it is consistent with studies showing that highly engaging distractor tasks can reduce pain perception in highly anxious individuals. ${ }^{6,38}$

This result contrasts with the studies from Arntz et al who found that anxiety-induced arousal did not significantly increase responses to a painful stimulus. ${ }^{16,72,73}$ However, Arntz's studies used state anxiety, whereas we investigated 
trait anxiety. Possibly, this difference explains the contrasting results of the effect of anxiety on pain perception. Our results are also challenged by the recent meta-analysis from Van Ryckeghem et $a{ }^{17}$ who found no effect of distraction or attention on pain perception. However, these studies were focused on chronic pain as opposed to the acute pain stimulus we used in our experiment, and there are many other studies that do show an effect of attentional strategies on acute pain perception. ${ }^{9}$

We did not observe an increase in pain perception in high anxious individuals in the distraction condition as we had predicted. Rather, distraction lowered pain perception across all participants in our study. This result is in line with findings by Schreiber et al, ${ }^{30}$ who showed greater distraction analgesia in high catastrophizing participants relative to low catastrophizing, and presumably less anxious, participants. While it is tempting to conclude that distraction tasks are more superior in attenuating pain experience regardless of individual differences, this conclusion cannot be made without the consideration of the following explanation. Studies have shown that cognitive engagement to a certain task depends not only on its cognitive characteristics (ie, cognitive load) but also on its affective characteristics. ${ }^{2,74}$ Interesting, novel, and pleasant tasks often motivate individuals to attend to the tasks, therefore capturing more attention and attenuating painful experience. ${ }^{75-78}$ This was illustrated in a study by Bantick et $a l^{38}$ in which they compared the distraction analgesia effect of a neutral and cognitively demanding task. They showed more reduction in pain intensity scores during the cognitively demanding task relative to the neutral distraction task. Similar results were reported by Johnson et al. ${ }^{6}$ These studies highlight the importance of the type of distraction task for producing an analgesic effect, especially in high anxious individuals, who are likely to have an attentional bias toward threats. ${ }^{28}$ Highly anxious individuals have difficulty disengaging their attention from the painful stimulus, and they would benefit most from a highly engaging distractor. However, distractors that are not sufficiently engaging are unlikely to attenuate pain, and these individuals are predicted to show an increase in pain perception.

Thus, although we predicted the highly anxious individuals would show an attentional bias to the threat and therefore have an increase in pain perception under the distraction condition, it is possible that the distractor used in this study is a game was sufficiently interesting and pleasant to distract even highly anxious individuals. Furthermore, the instruction to consume multi-colored pellets in order to increase the size of the snake may have motivated individuals to engage more cognitive resources to the distractor. As a result of the positive emotional and motivational valence of the distractor, emotional distress from painful stimulus was reduced in all participants, regardless of their pain-related trait anxiety level.

Furthermore, individuals engage in different strategies to alleviate the pain when painful stimuli are interpreted as threatening. ${ }^{79}$ Studies have shown that highly anxious individuals often engage in monitoring strategies, such as hypervigilance, to avoid impending pain. ${ }^{80}$ However, once pain is perceived, highly anxious individuals often engage in avoidance strategies, such as distracting themselves from the painful stimulus, to alleviate the experience of pain. ${ }^{80}$ Studies have suggested that matching individual's preferred coping strategy and attention strategy (eg, avoidance style with distraction task) is highly beneficial to alleviate laboratoryinduced pain. ${ }^{29,80}$ Hence, preference for avoidance coping style could explain the pain attenuation finding among highly anxious individuals in distraction group.

The current study demonstrated that attention can indeed influence pain perception. Specifically, focusing on a painful stimulus amplified pain perception while distraction attenuated pain perception. This suggests that engaging distractors are necessities that should not be absent in places such as hospital beds, ambulance, or emergency rooms, where experience of the pain is common. In addition, the current study found evidence showing that the influence of attention strategies on pain perception is dependent upon an individual's pain-related trait anxiety. Hence, medical practitioners should take this into consideration when they are designing a patient's pain management plan.

\section{Limitations and future directions \\ Content of information}

The current study was limited in the power of the anticipation manipulation. Although Group InfoExtra were provided with additional information about the expected discomfort, specific flow of the procedure, features of the equipment, and the safety of the procedure, ethical guidelines regarding human research required that participants in Group InfoControl to receive enough information about the painful stimulus and procedure to make an informed consent. Consequently, participants in both groups received similar information. These may account for why the manipulation of information was unsuccessful, which led to the non-significant difference in pain perception for both groups.

Although bound by the ethical restrictions, future studies should try to maximize the difference in quality and quantity 
of information provided to the two groups to better understand how information about impending pain affects pain perception. Furthermore, future studies may also include a manipulation check, such as asking participants to rate how threatening they perceive the experiment to be, to ensure that the manipulation of information was successful.

\section{Non-clinical sample}

It is also important to consider that the participants in this study comprised of healthy volunteers, and not clinically anxious individuals or chronically ill patients. Varying results may be attained if the participant pool consisted of clinical sample. Therefore, any extrapolation of the results from this study should be done with caution. Nonetheless, the current study serves as a stepping stone to expand the knowledge of pain alleviation methods that may help clinically anxious or chronically ill patients.

\section{Conclusion}

Pain is indeed a subjective experience that cannot be mitigated without understanding how this subjective experience arises. The current study examined potential factors that influence this subjective experience. Cognitive factors, such as attention, were found to be a potent component in influencing the perception of pain. Pain-related trait anxiety was found to moderate the effect that attention strategies have on pain perception. These results are indicative that assignment of an appropriate attention strategy can render extreme pain tolerable. Further explorations that address the aforementioned limitations should be done to unravel the remaining parts of the relationship, which may not only benefit individuals with acute pain but also individuals who suffer from chronic pain.

\section{Acknowledgments}

The authors would like to thank individuals and reviewers who have contributed their opinions, expertise, and support to this paper.

\section{Disclosure}

The authors report no conflicts of interest in this work.

\section{References}

1. Crombez G, Van Damme S, Eccleston C. Hypervigilance to pain: an experimental and clinical analysis. Pain. 2005;116(1-2):4-7.

2. Van Damme S, Legrain V, Vogt J, Crombez G. Keeping pain in mind: a motivational account of attention to pain. Neurosci Biobehav Rev. 2010;34(2):204-213.

3. Kahneman D. Attention and Effort. Vol. 1063. Englewood Cliffs, NJ: Prentice-Hall; 1973.
4. Eccleston C, Crombez G. Pain demands attention: a cognitiveaffective model of the interruptive function of pain. Psychol Bull. 1999;125(3):356-366.

5. Legrain V, Bruyer R, Guérit JM, Plaghki L. Involuntary orientation of attention to unattended deviant nociceptive stimuli is modulated by concomitant visual task difficulty. Evidence from laser evoked potentials. Clin Neurophysiol. 2005;116(9):2165-2174.

6. Johnson MH, Breakwell G, Douglas W, Humphries S. The effects of imagery and sensory detection distractors on different measures of pain: how does distraction work? Br J Clin Psychol. 1998;37(2):141-154.

7. Lautenbacher S, Pauli P, Zaudig M, Birbaumer N. Attentional control of pain perception: the role of hypochondriasis. J Psychosom Res. 1998;44(2):251-259.

8. Miron D, Duncan GH, Bushnell MC, Catherine Bushnell M. Effects of attention on the intensity and unpleasantness of thermal pain. Pain. 1989;39(3):345-352.

9. Kleiber C, Harper DC. Effects of distraction on children's pain and distress during medical procedures: a meta-analysis. Nurs Res. 1999;48(1): $44-49$.

10. Schmitt YS, Hoffman HG, Blough DK, et al. A randomized, controlled trial of immersive virtual reality analgesia, during physical therapy for pediatric burns. Burns. 2011;37(1):61-68.

11. Legrain V, Iannetti GD, Plaghki L, Mouraux A. The pain matrix reloaded. Prog Neurobiol. 2011;93(1):111-124.

12. Hadjipavlou G, Dunckley P, Behrens TE, Tracey I. Determining anatomical connectivities between cortical and brainstem pain processing regions in humans: a diffusion tensor imaging study in healthy controls. Pain. 2006;123(1-2):169-178.

13. Heinricher MM, Fields HL. Central Nervous System Mechanisms of Pain Modulation. Wall and Melzack's Textbook of Pain. 6th ed. 2013:129-142.

14. Tracey I, Ploghaus A, Gati JS, et al. Imaging attentional modulation of pain in the periaqueductal gray in humans. J Neurosci. 2002;22(7):2748-2752.

15. Valet M, Sprenger T, Boecker H, et al. Distraction modulates connectivity of the cingulo-frontal cortex and the midbrain during pain - an fMRI analysis. Pain. 2004;109(3):399-408.

16. Arntz A, Dreessen L, Merckelbach H. Attention, not anxiety, influences pain. Behav Res Ther. 1991;29(1):41-50.

17. Van Ryckeghem DM, Van Damme S, Eccleston C, Crombez G. The efficacy of attentional distraction and sensory monitoring in chronic pain patients: a meta-analysis. Clin Psychol Rev. 2018;59:16-29.

18. Rode S, Salkovskis PM, Jack T. An experimental study of attention, labelling and memory in people suffering from chronic pain. Pain. 2001;94(2): 193-203.

19. Villemure C, Bushnell CM. Cognitive modulation of pain: how do attention and emotion influence pain processing? Pain. 2002;95(3):195-199.

20. Sharpe L, Nicholson Perry K, Rogers P, Dear BF, Nicholas MK, Refshauge $\mathrm{K}$. A comparison of the effect of attention training and relaxation on responses to pain. Pain. 2010;150(3):469-476.

21. Chan SC, Chan CC, Kwan AS, Ting KH, Chui TY. Orienting attention modulates pain perception: an ERP study. PLoS One. 2012;7(6):e40215.

22. Keogh E, Hatton K, Ellery D. Avoidance versus focused attention and the perception of pain: differential effects for men and women. Pain. 2000;85(1-2):225-230.

23. Nouwen A, Cloutier C, Kappas A, Warbrick T, Sheffield D. Effects of focusing and distraction on cold pressor-induced pain in chronic back pain patients and control subjects. J Pain. 2006;7(1):62-71.

24. Apkarian AV, Baliki MN, Geha PY. Towards a theory of chronic pain. Prog Neurobiol. 2009;87(2):81-97.

25. Crombez G, Eccleston C, Van Damme S, Vlaeyen JW, Karoly P. Fear-avoidance model of chronic pain: the next generation. Clin J Pain. 2012;28(6): 475-483.

26. Kunz M, Chatelle $C$, Lautenbacher $S$, Rainville $P$. The relation between catastrophizing and facial responsiveness to pain. Pain. 2008;140(1): $127-134$. 
27. Leeuw M, Goossens ME, Linton SJ, et al. The fear-avoidance model of musculoskeletal pain: current state of scientific evidence. J Behav Med. 2007;30(1):77-94.

28. James JE, Hardardottir D. Influence of attention focus and trait anxiety on tolerance of acute pain. Br J Health Psychol. 2002;7(Pt 2):149-162.

29. Fox L, Walsh JC, Morrison TG, et al. Cognitive coping style and the effectiveness of distraction or sensation-focused instructions in chronic pain patients. PLoS One. 2016;11(4):e0142285.

30. Schreiber KL, Campbell C, Martel MO, et al. Distraction analgesia in chronic pain patients: the impact of catastrophizing. Anesthesiology. 2014;121(6):1292-1301.

31. Bar-Haim Y, Lamy D, Pergamin L, Bakermans-Kranenburg MJ, van Ijzendoorn MH. Threat-related attentional bias in anxious and nonanxious individuals: a meta-analytic study. Psychol Bull. 2007;133(1):1-24.

32. Cisler JM, Bacon AK, Williams NL. Phenomenological characteristics of attentional biases towards threat: a critical review. Cognit Ther Res. 2009;33(2):221-234.

33. Yiend J. The effects of emotion on attention: a review of attentional processing of emotional information. Cogn Emot. 2010;24(1):3-47.

34. Clarke PJ, Macleod C, Guastella AJ. Assessing the role of spatial engagement and disengagement of attention in anxiety-linked attentional bias: a critique of current paradigms and suggestions for future research directions. Anxiety Stress Coping. 2013;26(1):1-19.

35. Hadjistavropoulos HD, Hadjistavropoulos T, Quine A. Health anxiety moderates the effects of distraction versus attention to pain. Behav Res Ther. 2000;38(5):425-438.

36. Roelofs J, Peters ML, van der Zijden M, Vlaeyen JW. Does fear of pain moderate the effects of sensory focusing and distraction on cold pressor pain in pain-free individuals? J Pain. 2004;5(5):250-256.

37. Blitz B, Dinnerstein AJ. Role of attentional focus in pain perception: manipulation of response to noxious stimulation by instructions. $J$ Abnorm Psychol. 1971;77(1):42-45.

38. Bantick SJ, Wise RG, Ploghaus A, Clare S, Smith SM, Tracey I. Imaging how attention modulates pain in humans using functional MRI. Brain. 2002;125(Pt 2):310-319.

39. Atlas LY, Wager TD. How expectations shape pain. Neurosci Lett. 2012;520(2):140-148.

40. Grillon C, Baas JP, Lissek S, Smith K, Milstein J. Anxious responses to predictable and unpredictable aversive events. Behav Neurosci. 2004;118(5):916-924.

41. Foa EB, Zinbarg R, Rothbaum BO. Uncontrollability and unpredictability in post-traumatic stress disorder: an animal model. Psychol Bull. 1992;112(2):218-238.

42. Fonteyne R, Vervliet B, Hermans D, Baeyens F, Vansteenwegen D Reducing chronic anxiety by making the threatening event predictable: an experimental approach. Behav Res Ther. 2009;47(10):830-839.

43. Lin LY, Wang RH. Abdominal surgery, pain and anxiety: preoperative nursing intervention. J Adv Nurs. 2005;51(3):252-260.

44. Spalding NJ. Reducing anxiety by pre-operative education: make the future familiar. Occup Ther Int. 2003;10(4):278-293.

45. Bondy LR, Sims N, Schroeder DR, Offord KP, Narr BJ. The effect of anesthetic patient education on preoperative patient anxiety. Reg Anesth Pain Med. 1999;24(2):158-164.

46. Tang J, Gibson SJ. A psychophysical evaluation of the relationship between trait anxiety, pain perception, and induced state anxiety. J Pain. 2005;6(9):612-619.

47. Cornwall A, Donderi DC. The effect of experimentally induced anxiety on the experience of pressure pain. Pain. 1988;35(1):105-113.

48. Ozalp G, Sarioglu R, Tuncel G, Aslan K, Kadiogullari N. Preoperative emotional states in patients with breast cancer and postoperative pain. Acta Anaesthesiol Scand. 2003;47(1):26-29.

49. Staats PS, Staats A, Hekmat H. The additive impact of anxiety and a placebo on pain. Pain Med. 2001;2(4):267-279.

50. Vaughn F, Wichowski H, Bosworth G. Does preoperative anxiety level predict postoperative pain? AORN J. 2007;85(3):589-604.
51. Schofield P, Jefford M, Carey M, et al. Preparing patients for threatening medical treatments: effects of a chemotherapy educational DVD on anxiety, unmet needs, and self-efficacy. Support Care Cancer. 2008;16(1): 37-45.

52. Colloca L, Miller FG. The nocebo effect and its relevance for clinical practice. Psychosom Med. 2011;73(7):598.

53. Woo KY. Unravelling nocebo effect: the mediating effect of anxiety between anticipation and pain at wound dressing change. J Clin Nurs. 2015;24(13-14):1975-1984.

54. Miller K, Rodger S, Bucolo S, Greer R, Kimble RM. Multi-modal distraction using technology to combat pain in young children with burn injuries. Burns. 2010;36(5):647-658.

55. Klusman LE. Reducation of pain in childbirth by the alleviation of anxiety during pregnancy. J Consult Clin Psychol. 1975;43(2):162-165.

56. Giraudet-Le Quintrec J-S, Coste J, Vastel L, et al. Positive effect of patient education for hip surgery: a randomized trial. Clin Orthop Relat Res. 2003;414(414):112-120.

57. Brooks J, Tracey I. From nociception to pain perception: imaging the spinal and supraspinal pathways. J Anat. 2005;207(1):19-33.

58. Colloca L, Benedetti F. Nocebo hyperalgesia: how anxiety is turned into pain. Curr Opin Anaesthesiol. 2007;20(5):435-439.

59. Janssen SA. Negative affect and sensitization to pain. Scand J Psychol. 2002;43(2):131-137.

60. Thompson T, Keogh E, French CC. Sensory focusing versus distraction and pain: moderating effects of anxiety sensitivity in males and females. J Pain. 2011;12(8):849-858.

61. McCracken LM, Dhingra L. A short version of the pain anxiety symptoms scale (PASS-20): preliminary development and validity. Pain Res Manag. 2002;7(1):45-50.

62. Carleton RN, Asmundson GJ. The multidimensionality of fear of pain: construct independence for the fear of Pain Questionnaire-Short Form and the Pain Anxiety Symptoms Scale-20. J Pain. 2009;10(1):29-37.

63. Abrams MP, Carleton RN, Asmundson GJ. An exploration of the psychometric properties of the PASS-20 with a nonclinical sample. J Pain. 2007;8(11):879-886.

64. IBM SPSS Statistics for MAC [computer program]. Version 21.02012.

65. AMOS [computer program]. Version 21.02012.

66. Byrne BM. Structural Equation Modeling with AMOS: Basic Concepts, Applications, and Programming. Mahwah, NJ: Lawrence Erlbaum Associates; 2001.

67. Hooper D, Coughlan J, Mullen M. Structural Equation Modelling: Guidelines for Determining Model Fit. Electronic Journal of Business Research Methods. 2008;6(1),53-60.

68. Thibodeau MA, Welch PG, Katz J, Asmundson GJ. Pain-related anxiety influences pain perception differently in men and women: a quantitative sensory test across thermal pain modalities. Pain. 2013;154(3):419-426.

69. Johnson MH. How does distraction work in the management of pain? Curr Pain Headache Rep. 2005;9(2):90-95.

70. Moont R, Pud D, Sprecher E, Sharvit G, Yarnitsky D. "Pain inhibits pain" mechanisms: is pain modulation simply due to distraction? Pain. 2010;150(1):113-120.

71. Leventhal H. I know distraction works even though it doesn't! Health Psychol;11(4), 208-209.

72. ArntzA, de Jong P.Anxiety, attention and pain.JPsychosom Res. 1993;37(4): 423-431.

73. Arntz A, Dreessen L, de Jong P. The influence of anxiety on pain: attentional and attributional mediators. Pain. 1994;56(3):307-314.

74. Corbetta M, Shulman GL. Control of goal-directed and stimulus-driven attention in the brain. Nat Rev Neurosci. 2002;3(3):201-215.

75. Buck R, Morley S. A daily process design study of attentional pain control strategies in the self-management of cancer pain. Eur J Pain. 2006;10(5):385-398.

76. Hoffman HG, Seibel EJ, Richards TL, Furness TA, Patterson DR, Sharar SR. Virtual reality helmet display quality influences the magnitude of virtual reality analgesia. J Pain. 2006;7(11):843-850. 
77. Mitchell LA, MacDonald RA, Brodie EE. A comparison of the effects of preferred music, arithmetic and humour on cold pressor pain. Eur J Pain. 2006;10(4):343-351.

78. Rhudy JL, Williams AE, McCabe KM, Rambo PL, Russell JL. Emotional modulation of spinal nociception and pain: the impact of predictable noxious stimulation. Pain. 2006;126(1-3):221-233.
79. Vlaeyen JWS, Linton SJ. Fear-avoidance and its consequences in chronic musculoskeletal pain: a state of the art. Pain. 2000;85(3): 317-332.

80. Forys KL, Dahlquist LM. The influence of preferred coping style and cognitive strategy on laboratory-induced pain. Health Psychol. 2007;26(1):22-29.

\section{Publish your work in this journal}

The Journal of Pain Research is an international, peer reviewed, open access, online journal that welcomes laboratory and clinical findings in the fields of pain research and the prevention and management of pain. Original research, reviews, symposium reports, hypothesis formation and commentaries are all considered for publication.
Dovepress

The manuscript management system is completely online and includes a very quick and fair peer-review system, which is all easy to use. Visit http://www.dovepress.com/testimonials.php to read real quotes from published authors. 